\title{
Alcohol Demand, Delayed Reward Discounting, and Craving in Relation to Drinking and Alcohol Use Disorders
}

\author{
James MacKillop \\ University of Georgia and Brown University \\ Peter M. Monti \\ Providence Veterans Affairs Medical Center \\ and Brown University \\ James G. Murphy \\ University of Memphis and Brown University
}

\author{
Robert Miranda, Jr. \\ Brown University
}

\author{
Lara A. Ray \\ University of California, Los Angeles \\ and Brown University
}

\author{
Damaris J. Rohsenow, John E. McGeary, \\ and Robert M. Swift \\ Providence Veterans Affairs Medical Center \\ and Brown University
}

\author{
Jennifer W. Tidey and Chad J. Gwaltney \\ Brown University
}

\begin{abstract}
A behavioral economic approach to alcohol use disorders (AUDs) emphasizes both individual and environmental determinants of alcohol use. The current study examined individual differences in alcohol demand (i.e., motivation for alcohol under escalating conditions of price) and delayed reward discounting (i.e., preference for immediate small rewards compared to delayed larger rewards) in 61 heavy drinkers (62\% with an AUD). In addition, based on theoretical accounts that emphasize the role of craving in reward valuation and preferences for immediate rewards, craving for alcohol was also examined in relation to these behavioral economic variables and the alcohol-related variables. Intensity of alcohol demand and delayed reward discounting were significantly associated with AUD symptoms, but not with quantitative measures of alcohol use, and were also moderately correlated with each other. Likewise, craving was significantly associated with AUD symptoms, but not with alcohol use, and was also significantly correlated with both intensity of demand and delayed reward discounting. These findings further emphasize the relevance of behavioral economic indices of motivation to AUDs and the potential importance of craving for alcohol in this relationship.
\end{abstract}

Keywords: alcohol, behavioral economics, discounting, demand, craving

Behavioral economics integrates the principles of psychology and economics to understand how individuals make transactions with the world (Bickel et al., 2007; Camerer, 1999). The approach has been extensively applied to both normal and abnormal behavior, particularly in the area of alcohol use disorders (AUDs) and other substance use disorders (Vuchinich \& Heather, 2003). As it is applied to substance use, behavioral economics has made major contributions to characterizing how environmental factors, such as increases in cost or the presence of alternative reinforcers, affect alcohol and other substance use (Bigelow, Cohen, Liebson, \& Faillace, 1972; Higgins, Bickel, \& Hughes, 1994; Winger, Galuska, \& Hursh, 2007). Behavioral economics also recognizes the
James MacKillop, Department of Psychology, University of Georgia, and Center for Alcohol and Addiction Studies, Brown University; Robert Miranda, Jr., Jennifer W. Tidey, and Chad J. Gwaltney, Center for Alcohol and Addiction Studies, Brown University; Peter M. Monti, Damaris J. Rohsenow, John E. McGeary, and Robert M. Swift, Providence Veterans Affairs Medical Center, Providence, Rhode Island, and Center for Alcohol and Addiction Studies, Brown University; Lara A. Ray, Department of Psychology, University of California, Los Angeles, and Center for Alcohol and Addiction Studies, Brown University; James G. Murphy, Department of Psychology, University of Memphis, and Center for Alcohol and Addiction Studies, Brown University.

Lara A. Ray is now at the University of California, Los Angeles.
This research was partially supported by research and career grants from the Alcohol Beverage Medical Research Foundation (to James MacKillop), the National Institute on Alcohol Abuse and Alcoholism (AA 07850 to Peter M. Monti, AA 07459 to Lara A. Ray, AA 014966 to Robert Miranda, Jr., and AA 016936 to James MacKillop), and the Department of Veterans Affairs (to Peter M. Monti, Damaris J. Rohsenow, and John E. McGeary).

We are very grateful for the valuable research assistance of Amy Christian and John-Paul Massaro.

Correspondence concerning this article should be addressed to James MacKillop, Department of Psychology, University of Georgia, Athens, GA 30602. E-mail: jmackill@uga.edu 
importance of individual differences as contributors to substance use. As such, alcohol use and misuse are putatively a function of both individual characteristics (e.g., substance use history, conditioning history, decision-making biases, genetics), environmental factors (e.g., prices, consequences, alternative reinforcers), and the ongoing interaction of the two.

The individual-level variable that has been most extensively studied to date is variation in discounting of delayed rewards (i.e., preference for smaller immediate rewards versus larger delayed rewards), which is considered a behavioral economic index of impulsivity (Ainslie, 1975). Individuals who misuse alcohol have consistently demonstrated significantly steeper devaluation of delayed rewards than controls participants (Bjork, Hommer, Grant, \& Danube, 2004; Boettiger et al., 2007; Field, Christiansen, Cole, \& Goudie, 2007; J. M. Mitchell, Fields, D'Esposito, \& Boettiger, 2005; J. M. Mitchell, Tavares, Fields, D'Esposito, \& Boettiger, 2007; Petry, 2001; Vuchinich \& Simpson, 1998). This difference has also been evident in smokers (e.g., Baker, Johnson, \& Bickel, 2003), stimulant dependent individuals (e.g., Coffey, Gudleski, Saladin, \& Brady, 2003), opiate dependent individuals (Madden, Petry, Badger, \& Bickel, 1997), and pathological gamblers (e.g., MacKillop, Anderson, Castelda, Mattson, \& Donovick, 2006). From a clinical perspective, this precipitous devaluation of delayed rewards is theorized to be the basis for the repeated loss of control that characterizes addiction (Ainslie, 2001; Bickel \& Marsch, 2001). For example, an individual may report a preference for the larger delayed rewards associated with sobriety or moderation (employment, good health and family relationships) but shift preference to the smaller but immediate rewards associated with alcohol use (intoxication, stress reduction) when alcohol is immediately available. Consistent with this notion, several studies have found that different measures of delayed reward discounting prospectively predict alcohol and other drug treatment outcomes (Krishnan-Sarin et al., 2007; MacKillop \& Kahler, 2009; Tucker, Foushee, \& Black, 2008; Tucker, Vuchinich, Black, \& Rippens, 2006; Tucker, Vuchinich, \& Rippens, 2002; Yoon et al., 2007).

A second characteristic of increasing interest is variation in substance demand, or the quantitative relationship between consumption and cost (Hursh, Galuska, Winger, \& Woods, 2005). Demand is typically measured using demand curves, which characterize substance consumption over escalating levels of cost and provide several dimensions of the relative value of the substance. A demand curve prototypically decreases as a function of price, and the accompanying expenditure curve exhibits an inverted U-shaped curve. Across these two curves, each individual may vary on a number of indices of demand, including intensity (i.e., alcohol consumption at minimal cost), elasticity (i.e., slope of the demand curve in response to price), $P_{\max }$ (i.e., maximum inelastic price), $O_{\max }$ (i.e., maximum expenditure on alcohol), and breakpoint (i.e., the price that suppresses alcohol consumption to zero). Recent studies have suggested that these indices of alcohol demand are meaningfully related to alcohol use. Murphy and MacKillop (2006) found that heavy drinkers from a college sample exhibited significantly greater intensity, $O_{\max }$ (maximum expenditure), and breakpoint compared to light drinkers. Similarly, in a clinical application, MacKillop and Murphy (2007) found that variation on a number of indices of demand predicted response to a brief intervention for heavy drinking. In addition, meaningful variation in substance demand has been found among nicotine dependent and opiate dependent individuals (Greenwald \& Hursh, 2006; Jacobs \& Bickel, 1999).

Another area of increasing interest is in the role of craving from a behavioral economic perspective. From a theoretical standpoint, Loewenstein (1996) has argued that contrary to the widely held economic assumption of a purely rational agent, an individual's value (i.e., utility) for many commodities fluctuates substantially as a result of "visceral" factors, or powerful experiential motivational states. These effects are believed to apply to general commodities as well as alcohol and other drugs, although the nature of the visceral factor varies based on the commodity. For example, in the same way that the relative value of food is thought to vary as a function of hunger, the relative value of alcohol is theorized to increase as a function of craving for alcohol. A similar relationship has been proposed by Laibson (2001), with more emphasis on the role of environmental cues that signal rewards and robustly elicit potent increases in craving (Carter \& Tiffany, 1999). Craving is theorized to affect both the relative value of the commodity and more general reward-related processes like delayed reward discounting (Loewenstein, 1996). Although this approach has considerable potential for explaining the persistently excessive consumption of alcohol, tobacco, illicit drugs, and other appetitive targets, such as food, only a relatively small number of studies have been conducted in the area. In laboratory studies, craving for alcohol has been associated with subsequent choices for alcohol versus money (de Wit \& Chutuape, 1993; MacKillop, Menges, McGeary, \& Lisman, 2007), and a similar relationship has been observed for other substances (Badger et al., 2007; Perkins, Epstein, Grobe, \& Fonte, 1994; Sayette, Martin, Wertz, Shiffman, \& Perrott, 2001). In addition, enforced substance abstinence, which induces withdrawal and elicits craving, has been found to result in more impulsive discounting in smokers (Field, Santarcangelo, Sumnall, Goudie, \& Cole, 2006; S. H. Mitchell, 2004) and opiate dependent individuals (Giordano et al., 2002).

Taken together, two current foci in a behavioral economic approach to addictive behavior are to identify relevant individual difference variables and to integrate the role of craving. We sought to address both of these areas in the current study. The first objective was to examine delayed reward discounting and alcohol demand in relation to alcohol use and AUD severity, as well as in relation to each other. No previous studies have compared alcohol demand and discounting to each other. The second objective of the study was to directly examine craving for alcohol in relation to both the behavioral economic variables and alcohol-related variables. Previous studies have largely overlooked the specific role of craving in relation to these variables. On the basis of the existing literature and theoretical accounts, we predicted that the behavioral economic variables and craving would be significantly associated with AUD severity, above and beyond alcohol use itself, and that behavioral economic variables would be related to craving. Of the various indices of alcohol demand, on the basis of previous findings with college student drinkers, we specifically predicted that intensity of demand and $O_{\max }$ would exhibit the highest magnitude associations. 


\section{Method}

\section{Participants}

We recruited 61 participants (38\% women, $62 \%$ men) from the community using newspaper and bus advertisements, which solicited individuals who were 21-65 years old, regular drinkers, and not seeking treatment for alcohol problems (i.e., no interest in treatment for alcohol problems and no treatment within the preceding 90 days). Participants were assessed at the outset of a 5 -week study of the effects of an anticonvulsant medication on drinking and other variables (Miranda et al., 2008). For inclusion, individuals were required to drink 18-60 drinks per week (for men; 14-53 for women) and to be physically healthy as determined by a physical exam. Minimum drinking criteria were to recruit heavy drinkers and maximum drinking criteria were for safety purposes. Participants were also required not to use illicit drugs (determined via self-report and urine toxicology screen) and not to exhibit evidence of clinical depression (Beck Depression Inventory II score < 14; Beck, Steer, \& Brown, 1996). Participants were on average 42.4 years old $(S D=13.1) ; 88 \%$ were White, $5 \%$ were African American, 2\% were Native American, 5\% were biracial, and $10 \%$ were Hispanic (note that race and Hispanic ethnicity were not considered mutually exclusive). Participants' median income was $\$ 20,000-\$ 29,999$ per year. Over the previous three months, participants drank a mean of 29.09 drinks/week $(S D=13.60$; range $=10.19-68.79)$. This reflected a mean of $68 \%$ drinking days $(S D=22.51)$ and a mean of $42.25 \%$ heavy drinking days $(S D=28.50)$, defined as five drinks in an episode for men and four in an episode for women (National Institute on Alcohol Abuse and Alcoholism [NIAAA], 2005). Diagnostically, $62 \%$ ( $n=38 ; 34 \%$ women, $66 \%$ men) met criteria for an AUD (15\% abuse; $47 \%$ dependence). All individuals were heavy drinkers according to NIAAA (2005) guidelines. At no point over the course of the protocol did the research staff instruct participants to change their drinking.

\section{Procedure}

Assessments took place during individual in-person testing sessions. Participants were assessed for breath-alcohol level to ensure sobriety (Intoximeters Alco-Sensor IV; Intoximeters Inc., St. Louis, MO), and all demonstrated breath-alcohol levels of $0.00 \%$. Self-report measures were administered before the diagnostic interview, with the alcohol purchase task (measuring demand) prior to the Monetary Choice Questionnaire (measuring delayed reward discounting). All assessments were conducted at the Center for Alcohol and Addiction Studies at Brown University. All aspects of the study were approved by the institutional review board, and all participants provided informed consent (Miranda et al., 2008).

\section{Assessment}

Demographics. Participants completed a comprehensive demographics assessment, including sex, race, ethnicity, income, and other descriptive variables.

Alcohol use and AUD severity. Drinking during the 90 days prior to enrollment was assessed using the Timeline Followback (Sobell, Maisto, Sobell, \& Cooper, 1979), which has been vali- dated for assessing drinking during a circumscribed period of time when administered under the conditions of confidentiality and zero blood alcohol. Quantitative indices of alcohol use were drinks/ week, reflecting general volume of alcohol consumed, and percentage of heavy drinking episodes (five drinks in an episode for men and four in an episode for women; NIAAA, 2005), a drinking pattern that is strongly associated with negative consequences.

AUD severity was determined via the Structured Clinical Interview for DSM-Research Version (SCID; First, Gibbon, Spitzer, \& Williams, 1995), a semistructured interview that has been validated for assessing Diagnostic and Statistical Manual of Mental Disorders (4th ed.; DSM-IV) substance use disorders (Kranzler, Kadden, Babor, Tennen, \& Rounsaville, 1996). The AUDs and substance use disorders modules were administered by a trained doctoral-level clinical psychologist. The modules for both alcohol abuse and dependence were administered to all participants, and diagnosis was based on symptoms present in the last 12 months (American Psychiatric Association, 2000). AUD severity was operationalized as a symptom count from both the alcohol abuse and dependence SCID modules. A continuous approach was used because there is considerable evidence that alcohol problems are dimensional in nature and may be oversimplified when exclusively considered from a dichotomous or hierarchical standpoint (Kahler \& Strong, 2006; Ray, Kahler, Young, Chelminski, \& Zimmerman, 2008). The two AUD diagnoses have independent, nonoverlapping symptoms that were coded as being absent, subthreshold, or present.

Alcohol demand. Indices of alcohol demand were determined from an alcohol demand curve generated via an alcohol purchase task (APT), which has been validated in a number of studies (MacKillop \& Murphy, 2007; Murphy \& MacKillop, 2006). Participants were asked to estimate how many standard drinks they would consume in a typical drinking situation at an array of prices with the explicit conditions of no previous drinking and no alternative sources of alcohol. The instructions were as follows:

\footnotetext{
Please respond to these questions honestly, as if you were actually in this situation. Imagine that you are drinking in a TYPICAL SITUATION when you drink. The following questions ask how many drinks you would consume if they cost various amounts of money. The available drinks are standard size domestic beer $(12 \mathrm{oz}$.), wine $(5 \mathrm{oz}$.), shots of hard liquor $(1.5 \mathrm{oz}$.$) , or mixed drinks containing one shot of liquor.$ Assume that you did not drink alcohol before you are making these decisions, and will not have an opportunity to drink elsewhere after making these decisions. In addition, assume that you would consume every drink you request; that is, you cannot stockpile drinks for a later date or bring drinks home with you.
}

The instructional set was intentionally broad to be maximally applicable to the participants' diverse drinking patterns. The APT used 16 prices, ranging from no cost $(\$ 0)$ to $\$ 1,120$ per drink, based on previous validation studies (Jacobs \& Bickel, 1999; Murphy \& MacKillop, 2006).

Delayed reward discounting. Delayed reward discounting was assessed using the Monetary Choice Questionnaire (MCQ; Kirby, Petry, \& Bickel, 1999), a validated self-report measure of discounting. Individuals made 27 choices between smaller immediate rewards and larger delayed rewards that were preconfigured at various levels of hyperbolic discounting. The overall pattern of responding can be used to determine an estimate of their general 
temporal discounting function, commonly referred to as $k$, and temporal discounting of rewards at three levels of magnitude (small: \$25-\$35; medium: \$50-\$60; large: \$75-\$85). Participants in this study made choices for hypothetical rewards; a number of previous studies have found close correspondence between hypothetical and actual choices in delayed reward discounting paradigms (Lagorio \& Madden, 2005; Madden, Begotka, Raiff, \& Kastern, 2003; Madden et al., 2004).

Craving for alcohol. Craving for alcohol during the previous week was assessed using the Penn Alcohol Craving Scale (PACS; Flannery, Volpicelli, \& Pettinati, 1999), a psychometrically validated and unidimensional craving measure. The PACS exhibited high internal consistency in this sample $(\alpha=.89)$.

\section{Data Analysis}

All variables were initially screened for missing data, outliers (Zs > 3.29), and distribution abnormalities. Alcohol demand curves were modeled from the observed APT data and using Hursh and Winger's (1995) demand curve normalization equations. Elasticity for each participant was calculated via the following equations (Hursh \& Winger, 1995). Normalized dose $(q)$ was calculated as $q=100 / B$, where $B=$ consumption at the lowest price. Normalized dose was then used to generate values for normalized price $(P)$ as $P=F R / q$, where $F R$ is the response requirement, in this case, the price increment. Normalized dose was also used to generate values for normalized consumption $(Q)$ as $Q=R q$, where $R$ refers to reported consumption. These variables were then applied to Hursh and Winger's normalized demand equation, $\operatorname{Ln} Q=$ $\operatorname{Ln}(100)+b(\operatorname{Ln} P)-a P$, where $a$ and $b$ are derived parameters reflecting the initial slope and acceleration of the demand curve, respectively. Nonlinear regression was used to fit Hursh and Winger's normalized demand equation to the data and to generate an $R^{2}$ value, reflecting percentage of variance accounted for by the equation (i.e., the adequacy of the fit of the model to the data). Elasticity of demand was assessed at each price increment as $b-$ $a P$, and the overall level of elasticity (hereafter simply referred to as elasticity) was calculated as the mean of the individual price increment values. As with Jacobs and Bickel (1999), to permit the use of logarithmic transformations in the normalized demand equation, zero values for price without cost (free consumption) and breakpoint were replaced with arbitrarily low nonzero values (0.001). This effectively resulted in a minimum price of $\$ 0.001$ and a lowest unit of consumption of 0.001 drinks.

The additional facets of demand were generated using an observed values approach (Murphy \& MacKillop, 2006). Intensity was defined as free access consumption (i.e., alcohol consumption at zero cost). Breakpoint was defined as the first increment of cost at which no alcohol would be consumed. $O_{\max }$ was defined as the peak expenditure for alcohol. $P_{\max }$ was defined as the price associated with the peak expenditure. Delay discounting was calculated from the MCQ using the approach described by Kirby et al. (1999). Hyperbolic temporal discounting functions (i.e., $k$ ) were estimated based on each participant's array of responses overall and within the three magnitudes. Participants were assigned a $k$ value reflecting the highest consistency among the discounting values or the geometric mean of two or more $k$ values that were equally consistent (Kirby et al., 1999). As a validity check of the measure, a magnitude effect (i.e., greater discounting for smaller rewards than larger rewards) was examined using a within-subjects analysis of variance. Pearson's product-moment correlations and hierarchical linear regression were used to examine the continuous relationships among variables. Following zero-order correlations, hierarchical regressions were conducted to determine the unique contribution of the candidate variables in relation to AUD symptoms beyond quantitative indices of alcohol use and income. Covariates were entered into an initial block and the significance of the change in $R^{2}$ was used to determine an incremental contribution. Statistical significance for each independent variable was based on the significance of the regression coefficient. All analyses were conducted using SPSS Version 16.0.

\section{Results}

\section{Preliminary Analyses}

One subject did not complete the PACS and one subject did not complete the MCQ, but no other data were missing. A small number of outliers were identified $(<1 \%)$ but were determined to be legitimate values and were recoded as one unit above the next nonoutlying value (Tabachnick \& Fidell, 2001). Based on the distribution histograms, delay discounting and demand variables were positively skewed, as is common, and were logarithmically transformed, which improved the distributions, skewness, and kurtosis. To permit the transformation of elasticity, which is in negative units, the absolute value of elasticity was transformed and multiplied by -1 to retain its directionality. Demand curves were topographically prototypic, with consumption decreasing as a function of escalating price and expenditure conforming to an inverted U-shaped curve (see Figure 1). The normalized demand curve equation provided an excellent fit to the data (median $R^{2}=$ .91 , range $=.71-1.0$ ). Zero-order correlations among the behavioral economic variables, craving, and income are presented in Table 1. Consistent with previous findings, correlations among the indices of demand varied from very high to negligible. Correlations among the three magnitudes of discounting were very high, but a magnitude effect was also evident, $F(2,118)=41.58, p<$ $.001, \eta_{p}^{2}=.41$, reflecting greater discounting of smaller magnitude rewards. Discounting was consistently significantly associated with intensity of demand, but no other indices of demand.

\section{Demand, Discounting, and Craving in Relation to Drinking and AUD Severity}

Pearson's product-moment correlations were conducted between the behavioral economic and craving variables in relation to drinks/week, percent heavy drinking days (\%HDD), and AUD severity (see Table 1). Significant associations were evident between AUD symptoms and intensity of demand, all indices of discounting, and craving, but no significant associations were evident with drinks/week or \%HDD. Statistical trends $(p<.10)$ were observed between drinks/week and overall discounting and large discounting. Drinks/week and \%HDD were highly correlated with each other $(r=.82, p<.001)$, but only drinks/week was significantly correlated with AUD severity (drinks/week $r=.27$, $p<.05$; \%HDD $r=.07$ ). To illustrate these relationships, the 

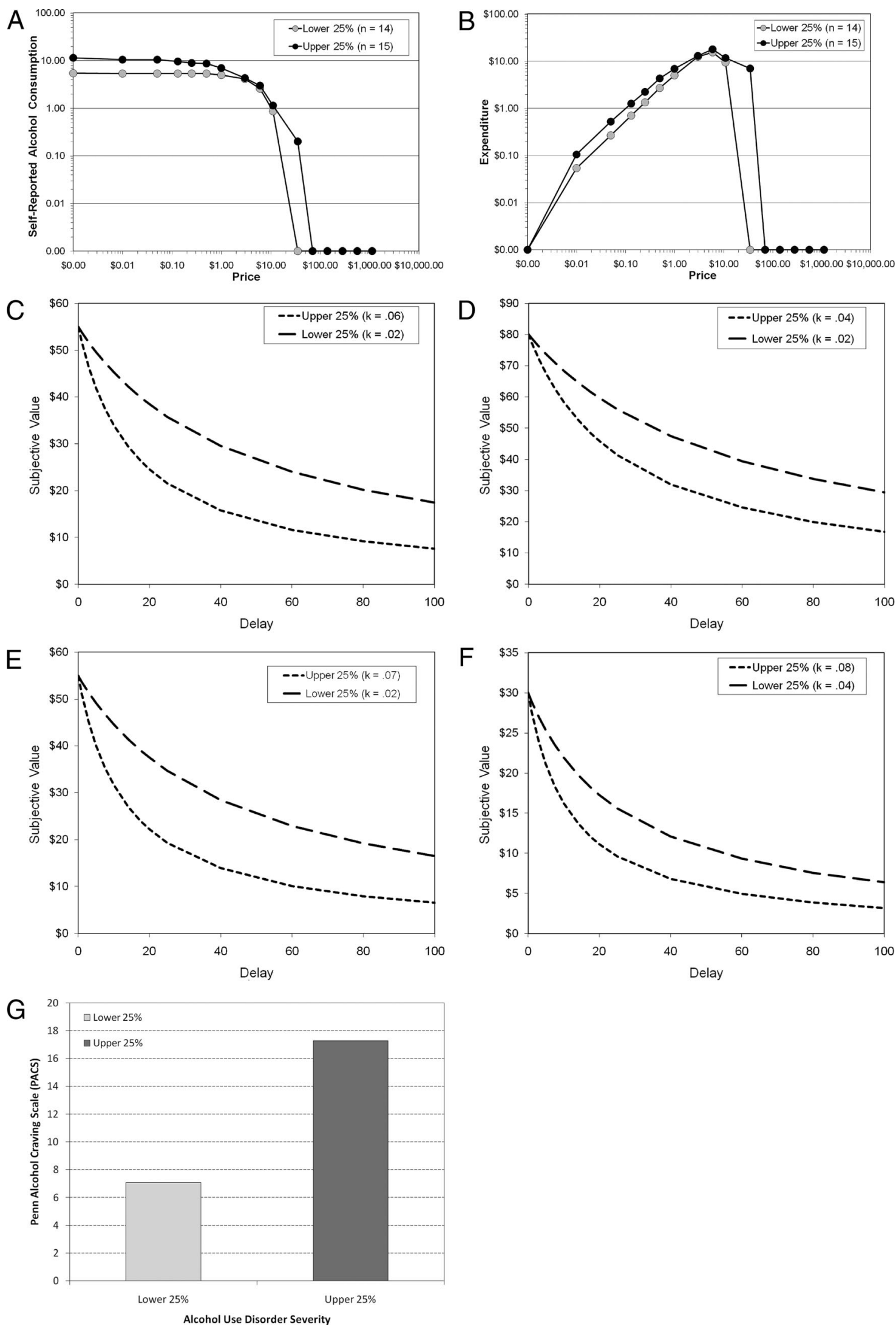

Figure 1 (opposite). 
Table 1

Correlations Among Facets of Alcohol Demand, Delayed Reward Discounting of Monetary Rewards, Craving, Annual Income, and Alcohol-Related Variables

\begin{tabular}{|c|c|c|c|c|c|c|c|c|c|c|c|c|c|c|}
\hline Variable & 1 & 2 & 3 & 4 & 5 & 6 & 7 & 8 & 9 & 10 & 11 & 12 & 13 & 14 \\
\hline 1. Intensity & - & & & & & & & & & & & & & \\
\hline 2. Elasticity & -.02 & - & & & & & & & & & & & & \\
\hline 3. $O_{\max }$ & $.33^{* *}$ & $.76^{* * * *}$ & - & & & & & & & & & & & \\
\hline 4. $P_{\max }$ & -.07 & $.73^{* * * *}$ & $.72^{* * * *}$ & - & & & & & & & & & & \\
\hline 5. Breakpoint & .08 & $.89^{* * * *}$ & $.78^{* * * * *}$ & $.82^{* * * *}$ & - & & & & & & & & & \\
\hline 6. Overall $k$ & $.34^{* * *}$ & .08 & -.03 & -.04 & -.05 & - & & & & & & & & \\
\hline 7. Large $k$ & $.30^{*}$ & .08 & -.10 & -.06 & -.06 & $.88^{* * * * *}$ & - & & & & & & & \\
\hline 8. Medium $k$ & $.44^{* * * *}$ & .11 & .00 & -.02 & -.06 & $.95^{* * * *}$ & $.84^{* * * *}$ & - & & & & & & \\
\hline 9. Small $k$ & $.28^{*}$ & .11 & .01 & -.01 & -.06 & $.89^{* * * * *}$ & $.73^{* * * *}$ & $.87^{* * * *}$ & - & & & & & \\
\hline 10. PACS & $.25^{*}$ & -.09 & .08 & .03 & .09 & $.23^{\dagger}$ & $.33^{* *}$ & $.22^{\dagger}$ & .13 & - & & & & \\
\hline 11. Income & $-.25^{*}$ & $.32^{*}$ & .19 & $.28^{*}$ & $.30^{* *}$ & $-.35^{* * *}$ & $-.37^{* * *}$ & $-.36^{* * *}$ & $-.32^{* *}$ & -.15 & - & & & \\
\hline 12. Drinks/week & .15 & .06 & .20 & .11 & .13 & $.23^{\dagger}$ & $.22^{\dagger}$ & .21 & .20 & -.21 & -.14 & - & & \\
\hline 13. \%HDD & .01 & -.01 & .17 & .14 & .04 & .08 & .01 & .06 & .13 & .12 & -.06 & $.82^{* * * * *}$ & - & \\
\hline 14. AUD severity & $.30^{*}$ & .01 & -.05 & -.15 & 01 & $.51^{* * * *}$ & $.50^{* * * *}$ & $.50^{* * * *}$ & $.41^{* * * *}$ & $.56^{* * * *}$ & $-.33^{* *}$ & $.28^{*}$ & .07 & - \\
\hline
\end{tabular}

Note. Correlations are Pearson product-moment correlations. Delayed reward discounting was examined overall as well as at three magnitudes (large, medium, and small). $O_{\max }=$ maximum output (expenditure); $P_{\max }=$ price maximum; $k=$ temporal discounting function; PACS $=$ Penn Alcohol Craving Scale; $\% \mathrm{HDD}=$ percent heavy drinking days; AUD $=$ alcohol use disorder.

${ }^{\dagger} p \leq .10 .{ }^{*} p \leq .05 .{ }^{* * *} p \leq .01$. $^{* * * *} p \leq .001$.

mean levels of demand, discounting, and craving in the upper and lower quartiles of AUD severity are depicted in Figure 1.

To clarify the unique associations with AUD severity, hierarchical regressions included a covariate block of drinks/week and income. In these models, \%HDD was not included because of its close correspondence with drinks/week ( $r=.82$; see Table 1$)$ and nonsignificant association with AUD severity. Intensity of demand was determined to make only a marginal incremental contribution to predicted variance (covariate model $\left.R^{2}=.16, \Delta R^{2}=.04\right), F(1$, $57)=2.82, p=.10$. In contrast, significant incremental contributions were evident for overall (covariate model $R^{2}=.16, \Delta R^{2}=$ $.15), F(1,56)=11.76, p<.001$, large (covariate model $R^{2}=.16$, $\Delta R^{2}=.14, F(1,56)=10.95, p<.005$, medium (covariate model $\left.R^{2}=.16, \Delta R^{2}=.14\right), F(1,56)=11.31, p<.001$, and small discounting (covariate model $\left.R^{2}=.16, \Delta R^{2}=.08\right), F(1,56)=$ $6.07, p<.05$. This was the case also for craving using the same covariate model (covariate model $\left.R^{2}=.16, \Delta R^{2}=.26\right), F(1$, $57)=24.90, p<.001$. The regression coefficients for all variables in the combined models are provided in Table 2.

\section{Discussion}

This study sought to characterize individual differences in alcohol demand, delayed reward discounting, and craving in relation to alcohol consumption and AUD severity in a sample of heavy drinkers of whom a substantial proportion met criteria for an AUD. The results revealed a number of relationships that were consistent with our predictions but others that were not. Significant associa- tions were evident between intensity of demand, all indices of discounting, and craving for alcohol in relation to AUD severity. Among the various magnitudes of discounting of delayed rewards, the strongest relationships were evident at higher levels of reward magnitude, which appeared to be because of consistently greater devaluation of delayed rewards of small amounts of money (i.e., a magnitude effect). The relationships were specific to AUD severity as indicated by the limited associations with the two quantitative measures of alcohol consumption and the demonstrated incremental contributions to associated variance in the hierarchical analyses. For the same reason, although income was related to the behavioral economic variables, the observed associations were not attributable to variation in income. However, the contributions beyond the covariate model varied considerably, ranging from negligible (intensity of demand) to moderate (small magnitude discounting) to substantial (overall, large, and medium magnitude discounting; craving). Specifically, beyond the covariate model of weekly alcohol use and income, the candidate variables accounted for an additional $4 \%-26 \%$ of associated variance. These findings largely support the study's broad hypothesis that these behavioral economic indices are significantly related to AUD severity.

The study's second objective was to examine the interrelationships among the behavioral economic variables and craving based on theoretical accounts that emphasize craving as a powerful contributor to value decision-making (Laibson, 2001; Loewenstein, 1996). Among the individual variables associated with AUD severity (i.e., intensity, discounting, and craving), significant as-

Figure 1. (opposite) Mean alcohol demand, delayed reward discounting, and craving for alcohol for individuals in the upper and lower $25 \%$ of alcohol use disorder severity. Panels A and B depict the demand and expenditure curves, plotted in conventional double logarithmic coordinates for proportionality and to accommodate large interprice intervals. Panels $\mathrm{C}-\mathrm{F}$ depict the estimated hyperbolic temporal discounting functions ( $k$ ) for the average amount of the delayed reward over a 100-day period for items in each array of responses $(\mathrm{C}=$ overall, $\mathrm{D}=1$ large, $\mathrm{E}=$ medium, $\mathrm{F}=$ small $)$. Panel $\mathrm{G}$ depicts craving for alcohol as measured by the Penn Alcohol Craving Scale. 
Table 2

Hierarchical Multiple Regressions Examining the Associations Between Behavioral Economic Indices and Craving in Relation to Alcohol Use Disorder Symptoms

\begin{tabular}{lrcc}
\hline \multicolumn{1}{c}{ Variable } & \multicolumn{1}{c}{$B$} & $S E$ & $\beta$ \\
\hline Intensity of demand & & & \\
$\quad$ Drinks/week & 0.09 & 0.05 & $.21^{\dagger}$ \\
Income & -0.57 & 0.28 & $-.25^{*}$ \\
$\quad$ Intensity & 4.61 & 2.75 & $.21^{\dagger}$ \\
Discounting & & & \\
$\quad$ Drinks/week & 0.07 & 0.05 & .16 \\
Income & -0.36 & 0.27 & -.16 \\
Overall $k$ & 3.88 & 1.13 & $.42^{* * *}$ \\
Drinks/week & 0.07 & 0.05 & .17 \\
Income & -0.35 & 0.28 & -.15 \\
Large $k$ & 3.83 & 1.16 & $.41^{* * * *}$ \\
Drinks/week & 0.08 & 0.05 & .17 \\
Income & -0.35 & 0.28 & -.15 \\
Medium $k$ & 3.76 & 1.12 & $.41^{* * * *}$ \\
Drinks/week & 0.08 & 0.05 & .19 \\
Income & -0.46 & 0.28 & -.20 \\
Small $k$ & 3.19 & 1.30 & $.31^{*}$ \\
Craving & & & .12 \\
Drinks/week & 0.06 & 0.05 & $-.24^{* *}$ \\
Income & -0.55 & 0.24 & $.52^{* * * *}$ \\
PACS & 0.52 & 0.11 & \\
\hline
\end{tabular}

Note. Unstandardized regression coefficients, standard errors, standardized regression coefficients, and coefficient statistical significance are presented. $k=$ temporal discounting function; PACS $=$ Penn Alcohol Craving Scale.

${ }^{\dagger} p \leq .10 .{ }^{*} p \leq .05$. ${ }^{* * *} p \leq .001$.

sociations were also observed among the variables themselves. Although these findings are cross-sectional, the current study provides initial direct evidence that craving is meaningfully related to the behavioral economic constructs of demand and discounting.

The current study extends a behavioral economic approach to AUDs in a number of ways. Consistent with previous studies of discounting and AUDs (Petry, 2001) and other addictive disorders (Bickel \& Marsch, 2001), delayed reward discounting was determined to be highly relevant to alcohol-related pathology. This is further evidence of a substantial decision-making bias toward immediate rewards at the expense of future benefits that is theorized to underlie the loss of control (i.e., inability to adhere to commitments to limit drinking or abstain in the presence of the immediate opportunity to drink) that characterize alcohol abuse and dependence (Ainslie, 2001; Vuchinich \& Heather, 2003). Moreover, there was evidence that craving for alcohol may play a role in this relationship, and this study provides provisional support for behavioral economic accounts that incorporate and emphasize the role of craving. From this perspective, decisionmaking is persistently biased and compromised by cravings that lead the individual to overvalue immediate small rewards in spite of future negative consequences (Loewenstein, 1996). For example, during treatment, an individual with alcohol dependence may report a strong desire to change their behavior and stop drinking in the absence of discriminative stimuli (e.g., people and situations) associated with alcohol use. However, following treatment, craving resulting from alcohol-related cues (e.g., Monti et al., 1987) or stress (e.g., Fox, Bergquist, Hong, \& Sinha, 2007) putatively biases the individual toward immediate short-term rewards (i.e., alcohol), at the cost of his or her sobriety and its associated long-term benefits. Thus, the delay discounting process is thought to be the basis for the preference reversal from abstinence to alcohol consumption commonly observed among individuals with AUDs in treatment. In this context, the current findings are consistent with previous studies that have found that drug withdrawal, which typically increases craving, also increases impulsive discounting (Field et al., 2006; Giordano et al., 2002).

Potentially important as these positive findings may be, it is also important to emphasize that the study revealed a number of negative findings that are worthy of discussion. Contrary to our predictions, other than intensity of demand, the other indices of demand were largely unrelated to alcohol use and AUD severity. This suggests that although alcohol consumption was generally price sensitive, its price sensitivity was largely unrelated to consumption patterns or problems with alcohol. These findings diverge from previous findings in a sample of college student drinkers (Murphy \& MacKillop, 2006), where most indices of demand were associated with alcohol consumption and problems. However, in contrast to the current study, the previous study used a self-report measure of alcohol problems, not a diagnostic interview, and a larger sample of younger drinkers with highly variable patterns of alcohol use (Murphy \& MacKillop, 2006). As such, it is both possible that the current findings are partially a function of power and restriction of range, or that demand is in fact less relevant to alcohol use and AUD severity among adults.

Another consideration is the nature of the task in the different samples. In purchase tasks, the costs of alcohol (price or response requirement) are fixed by the experimenter and the subject has a single modality of responses (level of consumption). As such, greater experimental control in this procedure is at the cost of some ecological validity. In the current study, the approach may have played a role in this study because, for heavy drinkers, many of whom had AUDs, escalating costs may motivate an array of alternative behaviors, such as drinking at home rather than in bars, switching to cheaper brands of alcohol, or buying alcohol in bulk. It is important to keep in mind that alcohol-seeking is highly fungible in the natural environment and the current study did not address alternative behaviors. Thus, it would be premature to conclude that the facets of alcohol demand, other than intensity, are unrelated to alcohol use and AUDs on the basis of these findings alone. Given the relative dearth of studies in this area, fully addressing this question will depend on examining alcohol demand in additional samples and potentially concurrently assessing how increases in price actuate alternative behaviors. Of note, one consistent finding across studies that is surprising is that elasticity of demand is not related to alcohol use or alcohol-related problems. This may be because, as indicated in Figure 1, demand is fundamentally curvilinear, initially generally flat during a period of inelasticity, then sloping when demand is elastic, and flat again at its terminus. Thus, elasticity, as a linear summary statistic of the demand curve, may be less informative than the other indices that reflect distinct portions of the demand and expenditure curves.

More generally, these findings should also be interpreted in the context of the study's strengths and weaknesses. Strengths include a sample that was well-characterized using validated measures in terms of alcohol use and AUD severity and the highly consistent findings within the study. Intensity of demand, discounting, and 
craving were significantly associated with AUD severity and each other, whereas the other indices of demand were highly correlated with one another, but not with intensity, discounting, and craving, with the exception of $O_{\max }$. Highly similar relationship patterns among the indices of demand have also been evident in a number of previous studies (e.g., Jacobs \& Bickel, 1999; Johnson \& Bickel, 2006; Madden \& Bickel, 1999; Murphy \& MacKillop, 2006). However, an important limitation of the study was that the design was cross-sectional, and thus the causal relationship among these variables cannot be unambiguously inferred from these data. It is plausible that these variables represent etiological factors contributing to the development an AUD among heavy drinkers, but it is equally possible that the observed findings are consequences of the development of an AUD or a combination of causal and consequential factors. Another consideration pertains to the assessment of the behavioral economic variables, which used hypothetical decision-making. Although previous studies have revealed close correspondence between choices for hypothetical and actual rewards (e.g., Lagorio \& Madden, 2005; Madden et al., 2004), it is also possible that using actual rewards would have revealed different results. Finally, it should be noted that there was not complete correspondence between the timeframes for drinking behavior (90 days) and AUD symptoms (12 months), which may have affected the observed associations.

To conclude, in the current study we sought to extend previous work using behavioral economics to understand alcohol misuse by examining alcohol demand, delayed reward discounting, and alcohol craving in relation to AUDs. The predictions were largely supported, particularly underscoring the importance of delayed reward discounting in relation to AUDs and providing evidence that craving may also play an important role in behavioral economic models of addiction. Although a number of considerations apply, this study extends a behavioral economic approach to AUDs and indicates the importance of incorporating the role of craving into this approach. Future studies will be necessary to further clarify these relationships.

\section{References}

Ainslie, G. (1975). Specious reward: A behavioral theory of impulsiveness and impulse control. Psychological Bulletin, 82, 463-496.

Ainslie, G. (2001). Breakdown of will. Cambridge, England: Cambridge University Press.

American Psychiatric Association. (2000). Diagnostic and statistical manual of mental disorders (4th ed., text rev.). Washington, DC: Author.

Badger, G. J., Bickel, W. K., Giordano, L. A., Jacobs, E. A., Loewenstein, G., \& Marsch, L. (2007). Altered states: The impact of immediate craving on the valuation of current and future opioids. Journal of Health Economics, 26, 865-876.

Baker, F., Johnson, M. W., \& Bickel, W. K. (2003). Delay discounting in current and never-before cigarette smokers: Similarities and differences across commodity, sign, and magnitude. Journal of Abnormal Psychology, 112, 382-392.

Beck, A., Steer, R., \& Brown, G. (1996). Manual for the Beck Depression Inventory-II. San Antonio, TX: Psychological Corporation.

Bickel, W. K., \& Marsch, L. A. (2001). Toward a behavioral economic understanding of drug dependence: Delay discounting processes. Addiction, 96, 73-86.

Bickel, W. K., Miller, M. L., Yi, R., Kowal, B. P., Lindquist, D. M., \& Pitcock, J. A. (2007). Behavioral and neuroeconomics of drug addiction:
Competing neural systems and temporal discounting processes. Drug and Alcohol Dependence, 90(Suppl. 1), S85-S91.

Bigelow, G., Cohen, M., Liebson, I., \& Faillace, L. A. (1972). Abstinence or moderation? Choice by alcoholics. Behaviour Research and Therapy, $10,209-214$.

Bjork, J. M., Hommer, D. W., Grant, S. J., \& Danube, C. (2004). Impulsivity in abstinent alcohol-dependent patients: Relation to control subjects and Type 1-/Type 2-like traits. Alcohol, 34, 133-150.

Boettiger, C. A., Mitchell, J. M., Tavares, V. C., Robertson, M., Joslyn, G., D'Esposito, M., \& Fields, H. L. (2007). Immediate reward bias in humans: Fronto-parietal networks and a role for the catechol- $O$ methyltransferase $158^{\mathrm{Val} / \mathrm{Val}}$ genotype. Journal of Neuroscience, 27, 14383-14391.

Camerer, C. (1999). Behavioral economics: Reunifying psychology and economics. Proceedings of the National Academy of Sciences, USA, 96, 10575-10577.

Carter, B. L., \& Tiffany, S. T. (1999). Meta-analysis of cue-reactivity in addiction research. Addiction, 94, 327-340.

Coffey, S. F., Gudleski, G. D., Saladin, M. E., \& Brady, K. T. (2003). Impulsivity and rapid discounting of delayed hypothetical rewards in cocaine-dependent individuals. Experimental and Clinical Psychopharmacology, 11, 18-25.

de Wit, H., \& Chutuape, M. A. (1993). Increased ethanol choice in social drinkers following ethanol preload. Behavioural Pharmacology, 4, 29-36.

Field, M., Christiansen, P., Cole, J., \& Goudie, A. (2007). Delay discounting and the alcohol Stroop in heavy drinking adolescents. Addiction, $102,579-586$.

Field, M., Santarcangelo, M., Sumnall, H., Goudie, A., \& Cole, J. (2006). Delay discounting and the behavioural economics of cigarette purchases in smokers: The effects of nicotine deprivation. Psychopharmacology, 186, 255-263.

First, M., Gibbon, M., Spitzer, R., \& Williams, J. (1995). User's guide for the Structured Clinical Interview for DSM-IV Axis I Disorders (SCID-I, Version 2.0.). New York State Psychiatric Institute, Biometrics Research Department.

Flannery, B. A., Volpicelli, J. R., \& Pettinati, H. M. (1999). Psychometric properties of the Penn Alcohol Craving Scale. Alcoholism: Clinical and Experimental Research, 23, 1289-1295.

Fox, H. C., Bergquist, K. L., Hong, K. I., \& Sinha, R. (2007). Stressinduced and alcohol cue-induced craving in recently abstinent alcoholdependent individuals. Alcoholism: Clinical and Experimental Research, 31, 395-403.

Giordano, L. A., Bickel, W. K., Loewenstein, G., Jacobs, E. A., Marsch, L., $\&$ Badger, G. J. (2002). Mild opioid deprivation increases the degree that opioid-dependent outpatients discount delayed heroin and money. Psychopharmacology, 163, 174-182.

Greenwald, M. K., \& Hursh, S. R. (2006). Behavioral economic analysis of opioid consumption in heroin-dependent individuals: Effects of unit price and pre-session drug supply. Drug and Alcohol Dependence, 85, $35-48$.

Higgins, S. T., Bickel, W. K., \& Hughes, J. R. (1994). Influence of an alternative reinforcer on human cocaine self-administration. Life Sciences, 55, 179-187.

Hursh, S. R., Galuska, C. M., Winger, G., \& Woods, J. H. (2005). The economics of drug abuse: A quantitative assessment of drug demand. Molecular Interventions, 5, 20-28.

Hursh, S. R., \& Winger, G. (1995). Normalized demand for drugs and other reinforcers. Journal for the Experimental Analysis of Behavior, 64, 373-384.

Jacobs, E. A., \& Bickel, W. K. (1999). Modeling drug consumption in the clinic using simulation procedures: Demand for heroin and cigarettes in opioid-dependent outpatients. Experimental and Clinical Psychopharmacology, 7, 412-426. 
Johnson, M. W., \& Bickel, W. K. (2006). Replacing relative reinforcing efficacy with behavioral economic demand curves. Journal for the Experimental Analysis of Behavior, 85, 73-93.

Kahler, C. W., \& Strong, D. R. (2006). A Rasch model analysis of DSM-IV Alcohol abuse and dependence items in the National Epidemiological Survey on Alcohol and Related Conditions. Alcoholism: Clinical and Experimental Research, 30, 1165-1175.

Kirby, K. N., Petry, N. M., \& Bickel, W. K. (1999). Heroin addicts have higher discount rates for delayed rewards than non-drug-using controls. Journal of Experimental Psychology: General, 128, 78-87.

Kranzler, H. R., Kadden, R. M., Babor, T. F., Tennen, H., \& Rounsaville, B. J. (1996). Validity of the SCID in substance abuse patients. Addiction, 91, 859-868

Krishnan-Sarin, S., Reynolds, B., Duhig, A. M., Smith, A., Liss, T., McFetridge, A., ... Potenza, M. N. (2007). Behavioral impulsivity predicts treatment outcome in a smoking cessation program for adolescent smokers. Drug and Alcohol Dependence, 88, 79-82.

Lagorio, C. H., \& Madden, G. J. (2005). Delay discounting of real and hypothetical rewards III: Steady-state assessments, forced-choice trials, and all real rewards. Behavioral Processes, 69, 173-187.

Laibson, D. (2001). The cue-theory of consumption. Quarterly Journal of Economics, 116, 81-119.

Loewenstein, G. (1996). Out of control: Visceral influences on behavior. Organizational Behavior and Human Decision Processes, 65, 272-292.

MacKillop, J., Anderson, E. J., Castelda, B. A., Mattson, R. E., \& Donovick, P. J. (2006). Divergent validity of measures of cognitive distortions, impulsivity, and time perspective in pathological gambling. Journal of Gambling Studies, 22, 339-354.

MacKillop, J., \& Kahler, C. W. (2009). Delayed reward discounting predicts treatment response for heavy drinkers receiving smoking cessation treatment. Drug and Alcohol Dependence, 104, 197-203.

MacKillop, J., Menges, D. P., McGeary, J. E., \& Lisman, S. A. (2007). Effects of craving and DRD4 VNTR genotype on the relative value of alcohol: An initial human laboratory study. Behavioral and Brain Functions, 3, 11

MacKillop, J., \& Murphy, J. G. (2007). A behavioral economic measure of demand for alcohol predicts brief intervention outcomes. Drug and Alcohol Dependence, 89, 227-233.

Madden, G. J., Begotka, A. M., Raiff, B. R., \& Kastern, L. L. (2003). Delay discounting of real and hypothetical rewards. Experimental and Clinical Psychopharmacology, 11, 139-145.

Madden, G. J., \& Bickel, W. K. (1999). Abstinence and price effects on demand for cigarettes: A behavioral-economic analysis. Addiction, 94, 577-588.

Madden, G. J., Petry, N. M., Badger, G. J., \& Bickel, W. K. (1997). Impulsive and self-control choices in opioid-dependent patients and non-drug-using control participants: Drug and monetary rewards. Experimental and Clinical Psychopharmacology, 5, 256-262.

Madden, G. J., Raiff, B. R., Lagorio, C. H., Begotka, A. M., Mueller, A. M., Hehli, D. J., \& Wegener, A. A. (2004). Delay discounting of potentially real and hypothetical rewards: II. Between- and withinsubject comparisons. Experimental and Clinical Psychopharmacology, 12, 251-261.

Miranda, R., Jr., MacKillop, J., Monti, P. M., Rohsenow, D. J., Tidey, J., Gwaltney, C., . . McGeary, J. (2008). Effects of topiramate on urge to drink and the subjective effects of alcohol: A preliminary laboratory study. Alcoholism: Clinical and Experimental Research, 32, 489-497.

Mitchell, J. M., Fields, H. L., D'Esposito, M., \& Boettiger, C. A. (2005). Impulsive responding in alcoholics. Alcoholism: Clinical and Experimental Research, 29, 2158-2169.

Mitchell, J. M., Tavares, V. C., Fields, H. L., D'Esposito, M., \& Boettiger, C. A. (2007). Endogenous opioid blockade and impulsive responding in alcoholics and healthy controls. Neuropsychopharmacology, 32, 439449 .
Mitchell, S. H. (2004). Effects of short-term nicotine deprivation on decision-making: Delay, uncertainty and effort discounting. Nicotine and Tobacco Research, 6, 819-828.

Monti, P. M., Binkoff, J. A., Abrams, D. B., Zwick, W. R., Nirenberg, T. D., \& Liepman, M. R. (1987). Reactivity of alcoholics and nonalcoholics to drinking cues. Journal of Abnormal Psychology, 96, 122-126.

Murphy, J. G., \& MacKillop, J. (2006). Relative reinforcing efficacy of alcohol among college student drinkers. Experimental and Clinical Psychopharmacology, 14, 219-227.

National Institute on Alcohol Abuse and Alcoholism. (2005). Helping patients who drink too much: A clinician's guide (NIH Publication No. 07-3769). U.S. Department of Health and Human Services, National Institutes of Health, National Institute on Alcohol Abuse and Alcoholism. Retrieved from http://pubs.niaaa.nih.gov/publications/Practitioner/ CliniciansGuide2005/guide.pdf

Perkins, K. A., Epstein, L. H., Grobe, J., \& Fonte, C. (1994). Tobacco abstinence, smoking cues, and the reinforcing value of smoking. Pharmacology Biochemistry Behavior, 47, 107-112.

Petry, N. M. (2001). Delay discounting of money and alcohol in actively using alcoholics, currently abstinent alcoholics, and controls. Psychopharmacology, 154, 243-250.

Ray, L. A., Kahler, C. W., Young, D., Chelminski, I., \& Zimmerman, M. (2008). The factor structure and severity of $D S M-I V$ alcohol abuse and dependence symptoms in psychiatric outpatients. Journal of Studies on Alcohol and Drugs, 69, 496-499.

Sayette, M. A., Martin, C. S., Wertz, J. M., Shiffman, S., \& Perrott, M. A. (2001). A multi-dimensional analysis of cue-elicited craving in heavy smokers and tobacco chippers. Addiction, 96, 1419-1432.

Sobell, L. C., Maisto, S. A., Sobell, M. B., \& Cooper, A. M. (1979) Reliability of alcohol abusers' self-reports of drinking behavior. Behaviour Research and Therapy, 17, 157-160.

Tabachnick, B. G., \& Fidell, L. S. (2001). Using multivariate statistics (4th ed.). Needham Heights, MA: Allyn \& Bacon.

Tucker, J. A., Foushee, H. R., \& Black, B. C. (2008). Behavioral economic analysis of natural resolution of drinking problems using IVR selfmonitoring. Experimental and Clinical Psychopharmacology, 16, 332340.

Tucker, J. A., Vuchinich, R. E., Black, B. C., \& Rippens, P. D. (2006) Significance of a behavioral economic index of reward value in predicting drinking problem resolution. Journal of Consulting and Clinical Psychology, 74, 317-326.

Tucker, J. A., Vuchinich, R. E., \& Rippens, P. D. (2002). Predicting natural resolution of alcohol-related problems: A prospective behavioral economic analysis. Experimental and Clinical Psychopharmacology, 10, $248-257$.

Vuchinich, R. E., \& Heather, N. (Eds.). (2003). Choice, behavioural economics and addiction. Amsterdam, the Netherlands: Pergamon/ Elsevier Science.

Vuchinich, R. E., \& Simpson, C. A. (1998). Hyperbolic temporal discounting in social drinkers and problem drinkers. Experimental and Clinical Psychopharmacology, 6, 292-305.

Winger, G., Galuska, C. M., \& Hursh, S. R. (2007). Modification of ethanol's reinforcing effectiveness in rhesus monkeys by cocaine, flunitrazepam, or gamma-hydroxybutyrate. Psychopharmacology, 193, 587598.

Yoon, J. H., Higgins, S. T., Heil, S. H., Sugarbaker, R. J., Thomas, C. S., \& Badger, G. J. (2007). Delay discounting predicts postpartum relapse to cigarette smoking among pregnant women. Experimental and Clinical Psychopharmacology, 15, 176-186.

Received July 15, 2008

Revision received June 5, 2009

Accepted June 5, 2009 